\title{
Meta
}

Journal des traducteurs

Translators' Journal

\section{Madeleine Moureau et Gerald Brace (2000) : Dictionnaires des sciences de la Terre/Comprehensive Dictionary of Earth \\ Science, anglais-français, français-anglais, Paris, Technip, Publications de l’Institut français du pétrole, xxix-1096 p.}

\section{André Clas}

Volume 45, numéro 4, décembre 2000

URI : https://id.erudit.org/iderudit/002276ar

DOI : https://doi.org/10.7202/002276ar

Aller au sommaire du numéro

Éditeur(s)

Les Presses de l'Université de Montréal

ISSN

0026-0452 (imprimé)

1492-1421 (numérique)

Découvrir la revue

Citer ce compte rendu

Clas, A. (2000). Compte rendu de [Madeleine Moureau et Gerald Brace (2000) : Dictionnaires des sciences de la Terre/Comprehensive Dictionary of Earth Science, anglais-français, français-anglais, Paris, Technip, Publications de l'Institut français du pétrole, xxix-1096 p.] Meta, 45(4), 676-679.

https://doi.org/10.7202/002276ar d'utilisation que vous pouvez consulter en ligne. 
Madeleine Moureau et Gerald Brace (2000) : Dictionnaires des sciences de la Terre/ Comprehensive Dictionary of Earth Science, anglais-français, français-anglais, Paris, Technip, Publications de l'Institut français du pétrole, Xxıx-1096 p.

Voici le troisième dictionnaire bilingue des deux mêmes auteurs. Comme les précédents (Dictionnaire des sciences et techniques du pétrole et Dictionnaire du forage et des puits), c'est encore, disons-le d'emblée, un ouvrage de la plus belle eau par les deux auteurs les plus compétents dans ce secteur et les plus riches d'expérience dans ce genre de recherche terminologique!

De même format, de même impression, de la même maison d'édition que les ouvrages précédents, c'est à nouveau un outil facile et agréable à manipuler : il tient bien dans la main, il est bien relié, bien imprimé sur du papier très blanc qui fait ressortir les entrées en caractères gras et rend la lecture et consultation aisées.

Le dictionnaire compte environ 37000 termes qui relèvent, comme l'indique le titre, des sciences de la Terre. Ce secteur de recherche devient, comme le signalent d'ailleurs les auteurs, de plus en plus multidisciplinaire et il n'est donc pas étonnant que l'ouvrage traite de trente-six domaines qu'il convient d'énumérer pour avoir une vue holistique de l'œuvre: archéologie, astronomie, biochimie, biologie, cartographie, climatologie, cristallographie, écologie, environnement, génétique, géochimie, géochronologie, géodésie, géologie, géomorphologie, géophysique, gîtologie, glaciologie, hydrogéologie, hydrologie, météorologie, minéralogie, océanographie, paléontologie, palynologie, pédologie, pétrographie, photogéologie, sédimentologie, sismologie, stratigraphie, taxinomie, tectonique, télédétection, topographie et volcanologie. 
Rappelons que l'étude des sciences de la Terre, comme l'écrit le professeur Dercourt, secrétaire perpétuel à l'Académie des sciences, dans sa Préface (p. v), «s'avèrent importantes pour le développement de nos sociétés modernes et la compréhension du monde qui nous entoure ». Comme chacun le sait, nous vivons sur et de la Terre et en tirons toutes nos ressources mais, il faut le signaler, nous n'avons pas tellement tenu compte de notre dépendance et nous l'avons souvent mise à mal et créé certains dysfonctionnements. Il nous faut à présent rapidement apprendre à mieux gérer notre potentiel terrestre et prendre conscience de l'importance de l'environnement et des primautés écologiques, dangereusement négligées sur notre planète, si nous voulons éviter les méfaits des variations climatiques et freiner la catastrophe écologique qui semble, à l'heure actuelle, s'annoncer de façon inéluctable. Le Dictionnaire vient donc bien à l'heure! La lexicographie et la terminographie contribuent, elles aussi, à la qualité de la vie par la compréhension des mots et des textes et par leur bonne utilisation!

L'établissement de la nomenclature d'un dictionnaire est toujours une tâche difficile et fort délicate, et couvrir de façon exhaustive trente-six domaines est une gageure impossible si on se limite à quelque 37000 termes. Les auteurs ont donc, comme il se doit, sélectionné des termes «sur la base de leurs occurrences dans les textes écrits et publiés dans chacune des deux langues»(p. Ix), méthode des plus rationnelles et des plus scientifiques. Le dictionnaire est ainsi proche de la réalité tangible et son utilité devient optimale. On ne s'étonnera donc pas si certains domaines sont mieux couverts que d'autres, mais les auteurs ont apporté une solution d'élargissement des plus astucieuses. C'est ainsi que les définitions fournies pour les entrées (en caractères gras), généralement un terme simple, ainsi que les entrées secondaires (en petits caractères gras) des mots composés et rangés dans un ordre alphabétique qui ne tient pas compte des articles et des prépositions, permettent facilement et sans grand risque de déduire la traduction d'autres termes composés, créant par cet enrichissement possible une nomenclature potentielle tout en obligeant à une certaine réflexion et à un dynamisme créateur très valorisant pour l'usager.

Ainsi, si le Dictionnaire donne « ablation - ablation $f$, perte de substance, enlèvement de matière; perte de matériaux d'un relief soumis à l'érosion mécanique ou chimique, elle s'exerce surtout sur les roches meubles et les sols dépourvus de couvert végétal; voir aussi glacial ablation» et donne ensuite comme entrées secondaires «ablation area: aire d'ablation [...], ablation moraine: moraine d'ablation [...], ablation rate: taux d'ablation $[\ldots]$ », il devient relativement facile de déduire les traductions, non répertoriées, d'ablation cone, ablation factor et ablation form, par exemple. Il faut en plus souligner la présence dans la nomenclature de certaines racines grecques et latines, signalées par un triangle, qui permettent de déduire à tout usager la signification de certaines formations. Ainsi, on a "acro- - acro-, du grec akros: extrême, extrémité», entrée qui permet de comprendre facilement le sens d' "acrocarpous - acrocarpe». En plus, ces entrées de racines, par exemple «batho- - batho-, ou bathy-, ou -bathe, du grec bathos: profond», donnent la signification de "batholith - batholite», répertoriés, mais autorisent aussi la traduction de acrobatholithic, terme de géologie non répertorié dans le dictionnaire.

Il faut encore signaler que les termes des entrées secondaires donnent pour certains substantifs, du moins dans la partie anglais-français, les composés qui ont comme deuxième terme le mot vedette. C'est ainsi que, par exemple, on trouvera sous 
"extinction - disparition $f$; extinction $f$ », en plus des termes dérivés extinction angle, extinction coefficient, extinction of a lake, extinction rate, également les formations mass extinction et mother of mass extinction. Le regroupement des formations avec le même mot vedette facilitent la recherche et permettent de voir l'ensemble des termes de la même «famille».

Pour éviter toute confusion quant à la question des majuscules et des minuscules, les auteurs suivent les principes suivants: seuls les noms de personnes, les noms géographiques dans les deux langues et les adjectifs correspondants en anglais, les noms des divisions chronostratigraphiques et géochronologiques, et les adjectifs correspondants en anglais, prendront une majuscule. On aura par exemple, pour les noms de personnes, "Kirchhoff - Gustav Robert Kichhoff (1824-1887), physicien allemand. Kirchhoff's law: loi de Kirchhoff», suivi de l'explication de la loi; ou, pour les noms géographiques, "Plaisancian - Plaisancien $m$, étage européen du Pléocène supérieur, équivalent de l'Astien; d'après Plaisance (Piacenza), ville du Nord de l'Italie».

Comme on a déjà pu le voir d'après les exemples cités, chaque terme est accompagné d'une traduction ou de plusieurs traductions, souvent d'une définition ou d'un commentaire et, s'il y a lieu, d'un renvoi qui apporte un complément d'information. S'il y a plusieurs traductions, elles sont données dans l'ordre alphabétique. Si le terme n'a pas de traduction, un commentaire ou un renvoi fournissent l'explication nécessaire. Les termes homographes ont chacun un article de dictionnaire particulier. Voyons quelques exemples:

chondrite - chondrite $f$; météorite non-différenciée, météorite primitive pierreuse très peu transformée depuis sa création au tout début du système solaire, ayant une texture spécifique en globule (chondrule); les chondrites forment $85 \%$ des météorites connues. avon - terme d'origine celtique désignant un cours d'eau; en Grande-Bretagne il se trouve souvent associé à des noms de lieux ou de rivières.

spouting - jaillissant(e), qui jaillit; se dit d'un liquide ou d'un fluide qui sort de terre de façon impétueuse. spouting hole: soufflard $m$. spouting spring: source jaillissante.

spouting - jaillissement $\mathrm{m}$.

stage - étage $m$, en stratigraphie, division chronostratigraphique de base; l'étage porte le nom de stratotype auquel on ajoute la désinence "ien" et s'écrit avec une majuscule; l'intervalle de temps pendant lequel un étage se forme est un âge; étape $f$, période au cours d'un processus; palier $m$; phase $f$; stade $m$, degré ou période qui dans une phase de développement peuvent être considérés comme une partie distincte. stage of evolution: stade d'évolution, un des états successifs d'un organisme, d'un sol, d'un relief. stage of maturity: stade de maturité, stade du cycle d'érosion où le creusement est moins actif. stage of early maturity: stade de début de maturité, d'un cycle d'érosion. stage of full maturity: stade de pleine maturité, d'un cycle d'érosion. stage of late maturity: stade de maturité avancée, d'un cycle d'érosion. stage of old age: stade final; stade de sénilité, stade du cycle d'érosion où le profil d'équilibre est atteint; stade de vieillesse. stage of youth: stade initial; stade de jeunesse, stade de creusement actif des rivières dans un cycle d'érosion. stage of early youth: stade de première jeunesse. stage of late youth: stade d'adolescence, d'un cycle d'érosion.

stage - platine $f$; plateau d'un instrument; table rigide d'un montage optique.

La partie anglais-français du dictionnaire est séparée de la partie français-anglais par 22 pages d'annexes de couleur vert pâle donnant en français et en anglais les règles d'écriture des grands nombres, les règles d'écriture des unités SI, les multiples et sous-multiples, la liste alphabétique des symboles des éléments naturels, les carac- 
téristiques physiques de la Terre, l'échelle des temps géologiques, l'échelle d'intensité des séismes, l'échelle de Beaufort, une table de conversion de température, la classification d'Homo sapiens et l'alphabet grec. Ces pages sont un condensé d'informations qu'il est sans nul doute utile d'avoir sous la main directement, comme pense-bête.

La partie français-anglais du dictionnaire est construite selon le même schème, mais avec bien entendu les définitions ou explications en langue cible, par exemple:

givre $\mathrm{m}$ - rime, opaque granular deposit of ice crystals on a solid surface caused by the freezing of supercooled fog droplets; silver frost, or silver thaw.

platier $\mathrm{m}$ - reef flat, top of a reef or coral and sandy expanse of reef that is generally dry at low tide. platier corallien : coral reef flat, area several hundred meters wide, composed of dead coral on top of which small colonies of live coral prosper and are uncovered at low tide. platier récifal: see platier. platier rocheux: rock bench, a wave-cut bench on a rock surface.

Les définitions en anglais ne sont pas la traduction des définitions en français, car elles proviennent de sources différentes, notamment des divers travaux de la Commission exploration du Comité des techniciens de la Chambre syndicale de la recherche et de la production du pétrole et du gaz naturel, de la Société géologique de France, de la Rand Corporation, de l'Université du Michigan, de diverses encyclopédies françaises et anglaises, de rapports de recherche, d'actes de congrès, etc. mais également de la consultation des nombreux spécialistes des sciences de la Terre.

Il convient d'adresser les félicitations aux auteurs pour la précellence de leur ouvrage. Ce nouveau dictionnaire est, à n'en pas douter, un superbe outil de référence: il est fiable, documenté, informatif, clair et dynamique, en ce sens qu'il stimule l'intérêt et développe la recherche de solutions par la créativité raisonnée. C'est un outil destiné aux spécialistes du domaine qu'il faut recommander aux enseignants, aux étudiants, aux chercheurs du domaine ou des domaines connexes. C'est encore un outil indispensable aux traducteurs qui doit faire partie de leur bibliothèque. C'est également un ouvrage indispensable au lecteur curieux qui cherche à mieux s'informer sur les sciences de la Terre, car il y trouvera une mine de renseignements et, sans nul doute, le point de départ vers des approfondissements sectoriels non négligeables. C'est finalement un ouvrage à recommander comme modèle d'analyse et de découverte méthodologiques dans les divers cours de formation en lexicographie et en terminologie. 